\title{
An Optimal Sensor Deployment Scheme to Ensure Multi Level Coverage and Connectivity in Wireless Sensor Networks
}

\author{
Arnab Raha ${ }^{1}$, Shovan Maity ${ }^{l}$, Mrinal Kanti Naskar ${ }^{l}$, Omar Alfandi $^{2}$, Dieter Hogrefe ${ }^{2}$ \\ ${ }^{1}$ Advanced Digital and Embedded Systems Laboratory, Dept. of ETCE, Jadavpur University. \\ arnabraha1989@gmail.com, shovanju35@gmail.com, mrinalnaskar@yahoo.co.in \\ ${ }^{2}$ Telematics Group, Institute of Computer Science, Georg August Universitaet Goettingen, Germany. \\ omar.alfandi@informatik.uni-goettingen.de, hogrefe@cs.uni-goettingen.de
}

\begin{abstract}
This paper introduces an optimal deployment algorithm of sensors in a given region to provide desired coverage and connectivity for a wireless sensor network. Our paper utilizes two separate procedures for covering different regions of a symmetrical rectangular area. The proposed method divides the given area of interest into two distinct sub-regions termed as the central and edge regions. In each region, a unique scheme is used to determine the number and location of sensors required to monitor and completely cover the region keeping the connectivity and coverage ranges of the sensors, their hardware specification and the dimensions of the region concerned as the constraints. Our scheme reduces the overhead in determining the position of sensors for deployment by following a coverage and connectivity algorithm of lesser complexity rather than those present in related schemes. Finally, we compare our deployment scheme with the interpolation scheme of [2] in regions of different dimensions and different coverage and connectivity levels with different sensing ranges of the sensors to show our cost efficiency over the latter.
\end{abstract}

Keywords-coverage, connectivity, WSN, k-coverage, kconnectivity, sensors, perimeter covered.

\section{INTRODUCTION}

WSNs form the face of modern communications. New techniques are frequently being developed to optimise the multi-faceted trade-offs between parameters like cost, connectivity, energy consumption, coverage, communication range and other application specific issues as needed.

A WSN refers to a collection of spatially and temporally distributed/used sensors i.e. devices that detects or measures a physical property and records and communicates it or responds to it. The entire set of nodes work as a unit reliably communicating with nearby nodes and completes the task they are expected to. Coverage and connectivity of wireless sensors are the parameters of our concern in this paper. Coverage refers to the sensing region of a node i.e. a sensor can detect a parameter only if it lies within the coverage of the concerned node. Connectivity refers to the range or area around a sensor node within which it can communicate reliably i.e. receive or transmit data to nodes or measure the concerned parameters within a certain degree of accuracy and precision. The collection of nodes deployed and working together in a given range forms a network. The network is said to be covered if every node has at least one other node within its coverage region. Equivalently, a network is connected if there is at least one path to exchange data between any two nodes in the network. A network is k-covered if every node has exactly $\mathrm{k}$ other nodes within its coverage region and k-connected if there is $\mathrm{k}$ distinct paths of data transmission between any two nodes.

Reliability of data transmission is a central issue in communication systems, typical examples being data generated in patient monitoring service in a hospital and military intelligence service in a battlefield. 1-coverage or 1connectivity is insufficient there because the breakdown of a single node or link may result in loss or corruption of important data. k-connectivity or $\mathrm{k}$-coverage (where $\mathrm{k} \geq 1$ ) and redundancy i.e. presence of duplicate nodes are needed here to ensure almost error free communication. But this improvement in efficiency is invariably accompanied by increased cost. Conversely, for tasks like daily temperature or rainfall sensing, cost is an important issue governing the deployment of sensors. Thus, an optimisation is desired which provides the maximum possible efficiency at minimum cost Such a design, despite ensuring multi-level coverage and connectivity is the aim of this paper.

\section{RELATED WORK}

Optimisation problems on coverage and connectivity are a topic of huge interest and practical applications in today's world. Efficient algorithms are frequently being developed in this field. The basic modelling of sensors and their architecture is discussed in [3] and [6]. [3] also addresses security issues \& schemes for protection of deployed sensor network from malicious attacks. Different studies have been done on the sensor implementation on the basis of specific protocols or standard parameters. Medium Access Control (MAC) layer protocol implementations for WSNs, Coverage Connectivity Protocols (CCP) and Special Purpose and Application Based Networks (SPAN) are discussed in [6].

Efficiency in power management is an issue of concern in handling sensors. Sensors mainly function in three modessleep (power saving) mode, listen (receiving) mode and talk (transmitting) mode. Optimization solutions deciding how much time a sensor spends in each mode, are addressed in [5] and [7].

Coverage and connectivity is the centre point of discussion for most WSNs. Geometrical and mathematical modelling of coverage and connectivity issues are there in [1], [4]-[7]. [5] even constricts the scope of discussion by assuming $r_{c} \geq 2 r_{s}$.

\begin{tabular}{|c|c|}
\hline Symbol & Meaning \\
\hline $\mathbf{r}_{\mathrm{s}}$ & Radius of sensing range(assumed circular in \\
& 2D) \\
\hline
\end{tabular}




\begin{tabular}{|l|l|}
\hline $\mathbf{r}_{\mathbf{c}}$ & Radius of communication range(circular) \\
\hline $\mathbf{k}_{\mathbf{s}}$ & $\begin{array}{c}\text { Level of connectivity(i.e. region is } \mathrm{k}_{\mathrm{s}}- \\
\text { connected) }\end{array}$ \\
\hline $\mathbf{k}_{\mathbf{c}}$ & \begin{tabular}{c} 
Level of coverage(i.e. region is $\mathrm{k}_{\mathrm{c}}$-covered) \\
\hline $\mathbf{d}$
\end{tabular} \\
$\begin{array}{c}\text { Distance between adjacent sensors in a row } \\
\text { (or column) }\end{array}$ \\
\hline
\end{tabular}

Reliability of coverage and connectivity i.e. multi-level coverage and connectivity is the prime point of discussion in our paper. [2], [7] discuses k-coverage for $\mathrm{k} \geq 1$ while [4] talks about full coverage with 2-connectivity and 1-connectivity. Our paper provides a generalised algorithm for both kcoverage and k-connectivity implementation at low cost.

\section{PROPOSED WORK}

\section{Problem statement:}

Given a region $\mathrm{R}$ we want to find an optimum deployment scheme so that the entire region is $\mathrm{k}_{\mathrm{s}}$ connected and $\mathrm{k}_{\mathrm{c}}$ covered. We assume the binary sensing model and propose our placement solutions. But first we describe the two placement solutions that have been proposed so far [2]:

\section{PAST WORK}

\section{A. The Duplicate Placement Scheme}

A simple way to achieve $\mathrm{k}$-coverage is to ensure 1-coverage and connectivity and then deploy $\mathrm{k}$ identical sensors at each designated location. For 1-coverage placement, [2] adopts the method proposed in [8], which uses the minimum number of sensors to achieve 1-coverage and connectivity [4]. It suggests placing sensors row by row, where each row of sensors guarantees continuous coverage and connectivity while adjacent rows guarantee continuous coverage of the whole area. According to the relation between $r_{c}$ and $r_{s}$, [8] divides the discussion into two cases:

1. When $r_{c}<\sqrt{3} r_{s}$, sensors on each row are separated by a distance of $r_{c}$, so the connectivity of sensors in each row is guaranteed. Also, each row of sensors covers a belt-like area of width $2 \alpha$, where $\alpha=\sqrt{ }\left(\mathrm{r}_{\mathrm{s}}{ }^{2}-.25 \mathrm{r}_{\mathrm{c}}{ }^{2}\right)$ Adjacent rows are shifted vertically by a distance $\left(\mathrm{r}_{\mathrm{s}}+\alpha\right)$, and horizontally by a distance of $r_{c} / 2$. This guarantees the coverage of the whole area.

2. When $r_{c}>\sqrt{3} r_{s}$, the above scheme uses a lot of extra sensors. So a regular placement of triangular lattice [9] is adopted, where adjacent sensors are separated by a distance of $\sqrt{ } 3 r_{s}$.

After determining the locations of the sensors by above methods they place $\mathrm{k}$ sensors at each location to achieve $\mathrm{k}$ coverage of the entire region. In the $1^{\text {st }}$ case, the distance between adjacent rows can be greater than $r_{c}$ so an extra column of sensors is necessary to connect the network where the distance between the sensors is less than or equal to $r_{c}$.

\section{B. The Interpolating Scheme}

Unlike the previous scheme, duplicate sensors are not used here. In this method, additional rows of sensors are deployed between original rows of sensors used to achieve 1-coverage using the method proposed in [8]. The deployment scheme is divided into 3 parts in [8] according to the relationship between $r_{c}$ and $r_{s}$.

Case I: $r_{c} \leq(\sqrt{ } 3 / 2) r_{s}$

The sensors are deployed to achieve 1-coverage as mentioned before. An additional row of sensors are added between each pair of adjacent rows to achieve 3-coverage. Each row is placed a distance $r_{\mathrm{s}}$ above the previous row. Adjacent sensors in each row are separated by a distance $r_{c}$. In this case, distance between adjacent rows is $r_{s}$. One more column of sensors separated by a distance of $r_{c}$ is deployed to connect adjacent rows.

Case II: $(\sqrt{ } \mathbf{3} / 2) \mathbf{r}_{\mathrm{s}}<\mathbf{r}_{\mathrm{c}} \leq(\mathbf{2}+\sqrt{ } \mathbf{3}) \mathrm{r}_{\mathrm{s}} / \mathbf{3}$

For 2-coverage, the previous scheme is used.Therefore, if the desired $\mathrm{k}=3$, we need to add one extra row of sensors between each new row and the corresponding old row. Each extra row is shifted horizontally by a distance $r_{c} / 2$ from the previous row. Neighbouring sensors are separated by a distance of $2 r_{c}$.

\section{Case III: $\mathbf{r}_{\mathrm{c}}>(2+\sqrt{ } \mathbf{3}) \mathbf{r}_{\mathrm{s}} / \mathbf{3}$}

Their scheme uses a strip based deployment scheme in which d is a certain fraction of $r_{s}$, depends on the desired level of coverage and connectivity and also on $r_{c}$.

\section{PRESENT WORK}

\section{Our Proposed deployment scheme}

We use a different deployment scheme for the edges and the central areas of the region to be monitored. Edges are defined as regions whose dimensions are $\leq \sqrt{2} \mathrm{r}_{\mathrm{s}}$ (proof for this is given later).

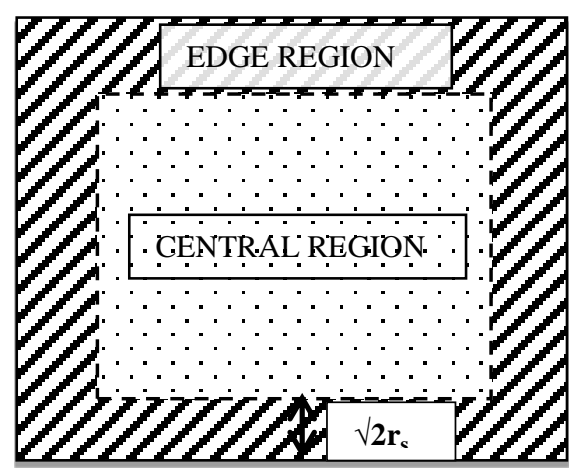

Fig.1. Demarcation between central and edge regions Step 1: Partition of the whole region

The entire region is divided into central and edge regions.

Step 2: Deployment in the Central regions

We divide our scheme into two cases dependent on $r_{c}$ and $r_{s}$ for central regions.

Case I: $\mathbf{r}_{\mathbf{c}} \geq \mathbf{r}_{\mathrm{s}}$

Adjacent sensors in a row (or column) are separated by a fixed distance $\mathbf{d}$ as found for different levels of coverage. $\mathrm{k}_{\mathrm{s}}=\mathrm{k}_{\mathrm{c}}$ here. Case II: $\mathbf{r}_{\mathbf{c}}<\mathbf{r}_{\mathrm{s}}$

Adjacent sensors are separated by a distance min $\left(r_{c}, \mathbf{d}\right)$ along the shorter dimension and a distance $\mathbf{d}$ along the longer dimension, d deduced just as in Case I. Sensors along the longer dimension are separated by a distance ' $d$ ' if $\min \left(r_{c}, \mathbf{d}\right)=$ d. However, if $\min \left(r_{c}, \mathbf{d}\right)=r_{c}$, we have to add ' $n$ ' additional rows or columns of sensor (depending on whether shorter dimension above=row or column) to achieve ' $n$ ' connectivity. Otherwise the level of connectivity is the same as the level of coverage as in Case I.

Step 3: Calculation of $d$ :

We use a $\mathrm{O}(n \log n)$ algorithm as in [1] for calculating the coverage level of a sensor's sensing region and the distance $\mathbf{d}$ 
for different levels of coverage and connectivity. We use a basic theorem stated in [1]:

Suppose that no two sensors are located in the same location. The whole network area is k-covered iff each sensor in the network is k-perimeter-covered. A sensor $\mathrm{s}_{\mathrm{i}}$ is said to be $\mathrm{k}$ perimeter-covered if all points on the perimeter of $s_{i}$ are perimeter-covered by at least $\mathrm{k}$ sensors other than $\mathrm{s}_{\mathrm{i}}$ itself.

We consider two sensors $s_{i}$ and $s_{j}$ located in positions $\left(x_{i}, y_{i}\right)$ and $\left(\mathrm{x}_{\mathrm{j}}, \mathrm{y}_{\mathrm{j}}\right)$ respectively. We denote the distance between the nodes by $\mathrm{d}_{(\mathrm{i}, \mathrm{j})}=\sqrt{ }\left(\mathrm{x}_{\mathrm{i}}-\mathrm{x}_{\mathrm{j}}\right)^{2}+\left(\mathrm{y}_{\mathrm{i}}-\mathrm{y}_{\mathrm{j}}\right)^{2}$ [Fig 2]. From simple geometry, $\angle \alpha=\arccos \left(\mathrm{d}_{(\mathrm{i}, \mathrm{j})} / 2 \mathrm{r}_{\mathrm{s}}\right)$. So arc of $\mathrm{s}_{\mathrm{i}}$ perimeter-covered by $\mathrm{s}_{\mathrm{j}}$ is $[\beta-$ $\alpha, \beta+\alpha]$.Here $\beta$ is the angle of the line joining $s_{i}$ and $s_{j}$ with respect to the horizontal.

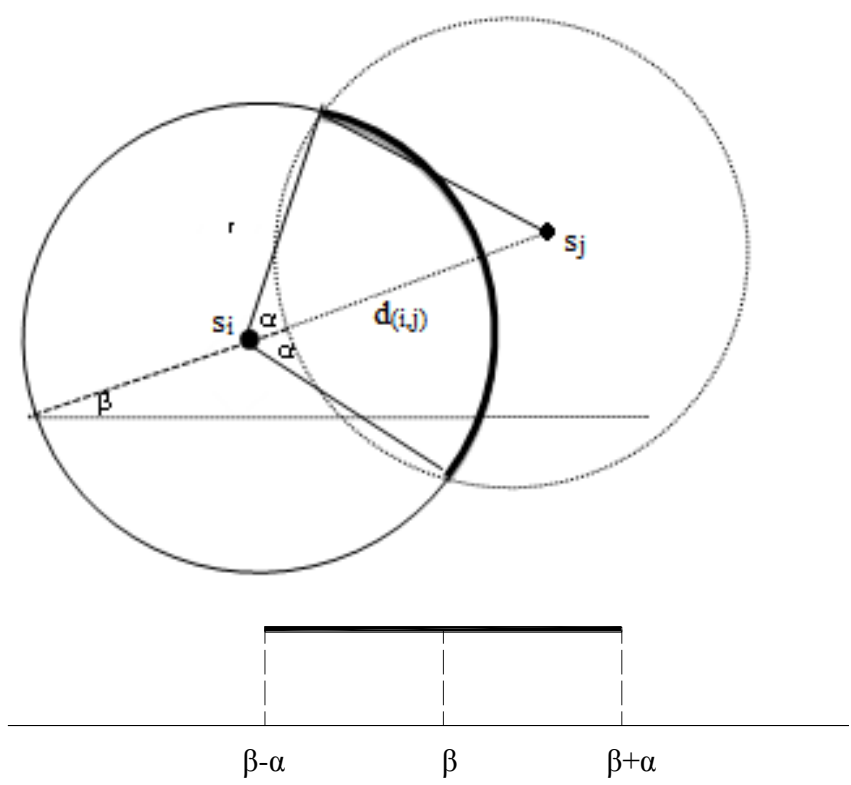

Fig.2: Angle contribution of $\mathrm{s}_{\mathrm{i}}$ for perimeter covered portion

Procedure for finding $d$ :

$$
\text { by } s_{j}
$$

In calculating $\mathbf{d}$ we consider only the k-perimeter covered problem for the $1^{\text {st }}$ quarter $\left[0^{\circ}\right.$ to $\left.90^{\circ}\right]$ of the sensing region of each node. As the deployment scheme is identical for all quadrants, the results are duplicated over quadrants 2,3 and 4.

A perimeter covered sensor has contributions mainly from 5 types of neighbouring sensors as shown in Fig 3 and defined in the tables below.

\begin{tabular}{|c|c|}
\hline Symbol & Meaning \\
\hline $\mathrm{S}_{\mathrm{i}}$ & i th sensor \\
\hline $\mathrm{x}_{\mathrm{i}}, \mathrm{y}_{\mathrm{i}}$ & $\mathrm{x}$ and $\mathrm{y}$ coordinates of $\mathrm{s}_{\mathrm{i}}$ \\
\hline $\mathrm{d}_{(\mathrm{i}, \mathrm{j})}$ & Distance between $s_{i}$ and $s_{j}$ \\
\hline$\alpha$ & $\begin{array}{l}\text { Half angular stretch of defined circles (of } \\
\text { radius } r \text { ) for intersection of } s_{i} \text { and } s_{j} \text { (from } \\
\text { centre of either node. Fig 2) }\end{array}$ \\
\hline$\alpha_{\mathrm{k}}$ & $\alpha$ for a sensor of type $\mathrm{k}(\mathrm{k}=1,2,3 \ldots, \mathrm{Fig} 3)$ \\
\hline$\beta$ & $\begin{array}{l}\text { Angle of the line joining } s_{i} \text { and } s_{j} \text { with respect } \\
\text { to the horizontal. }\end{array}$ \\
\hline $\mathrm{p}$ & $\begin{array}{l}\text { Subtype of each node in the upper right } \\
\text { coordinate }\end{array}$ \\
\hline q & Subtype of each node in the upper left \\
\hline
\end{tabular}

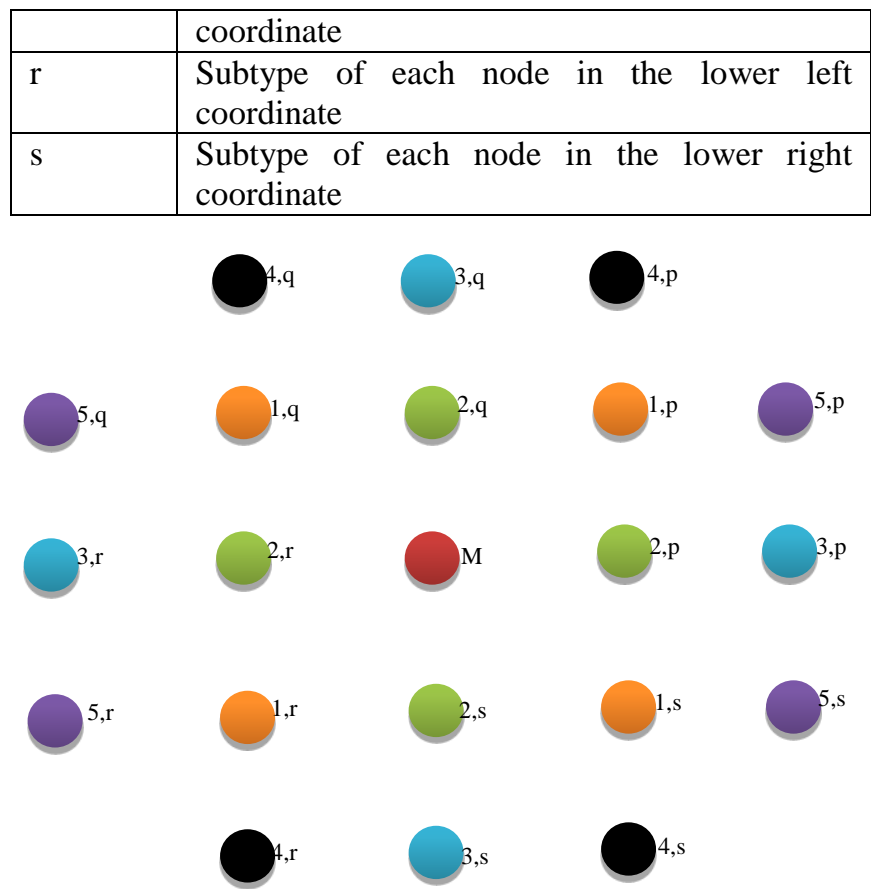

Fig.3: Types of sensors in coverage of the Main sensor

\begin{tabular}{|l|l|l|l|}
\hline $\begin{array}{l}\text { Symbol } \\
\text { Colors }\end{array}$ & Description of sensor & Type & $\mathbf{d}_{(\mathrm{i}, \mathrm{j})}$ \\
\hline $\mathbf{M}$ & Main Node & - & - \\
\hline 1 & Diagonal neighbours & 1 & $\mathrm{~V} 2 \mathrm{~d}$ \\
\hline 2 & $\begin{array}{l}\text { Row and column wise nearest } \\
\text { neighbour }\end{array}$ & 2 & $\mathrm{~d}$ \\
\hline 3 & $\begin{array}{l}\text { Row and column wise } 2 \mathrm{nd} \\
\text { nearest neighbour }\end{array}$ & 3 & $2 \mathrm{~d}$ \\
\hline $\mathbf{4}$ & $\begin{array}{l}\text { 2d distance column wise and } \\
\mathbf{d} \text { distance row wise }\end{array}$ & 4 & $\mathrm{~V} 5 \mathrm{~d}$ \\
\hline 5 & $\begin{array}{l}\mathbf{d} \text { distance column wise and } \\
\text { 2d distance row wise }\end{array}$ & 5 & $\mathrm{~V} 5 \mathrm{~d}$ \\
\hline
\end{tabular}

\#All sensor descriptions are with reference to M. Type and distance $\left(d_{(i, j)}\right)$ of each type of sensor is with reference to $M$. Simple geometry gives these results.

\section{Calculation of $\mathbf{d}$ for 2-coverage:}

Here, the perimeter of $\mathbf{s}$ is covered by nodes of type 1 and type 2.

For node of type $1 \mathrm{~d}_{(\mathrm{i}, \mathrm{j})}=\sqrt{ } 2 \mathrm{~d}$. So $\alpha_{1}=\arccos \left(\sqrt{ } 2 \mathrm{~d} / 2 \mathrm{r}_{\mathrm{s}}\right)$.

For node $1 \mathrm{p} \beta=45^{\circ}$. So arc of $\mathrm{M}$, perimeter covered by node of $1 p$ is $\left[45^{\circ}-\alpha_{1}, 45^{\circ}+\alpha_{1}\right]$.

For node of type $2 \mathrm{~d}_{(\mathrm{i}, \mathrm{j})}=\mathrm{d}$. So $\alpha_{2}=\arccos \left(\mathrm{d} / 2 \mathrm{r}_{\mathrm{s}}\right)$.

For node $2 \mathrm{p} \beta=0^{\circ}$. So arc of $\mathrm{M}$, perimeter covered by node $2 \mathrm{p}$ is $\left[-\alpha_{2}, \alpha_{2}\right]$. From the figure we see that sensor $M$ is 2-perimeter covered if arc of $\mathrm{M}$ perimeter covered by sensor $1 \mathrm{q}$ and sensor $1 \mathrm{p}$ overlap.

Range of perimeter coverage by node $1 \mathrm{q}$ is $\left[135^{\circ}-\alpha_{1}, 135^{\circ}+\alpha_{1}\right]$. So for 2 coverage $\left(135^{\circ}-\alpha_{1}\right)=\left(45^{\circ}+\alpha_{1}\right)$. Thus $\alpha_{1}=45^{\circ}$.

This gives us $\arccos \left(\sqrt{ } 2 \mathrm{~d} / 2 \mathrm{r}_{\mathrm{s}}\right)=45^{\circ}$ or $\mathrm{d}=\mathrm{r}_{\mathrm{s}}$.

For 2 coverage the distance between adjacent rows and columns is $d=r_{s}$. 


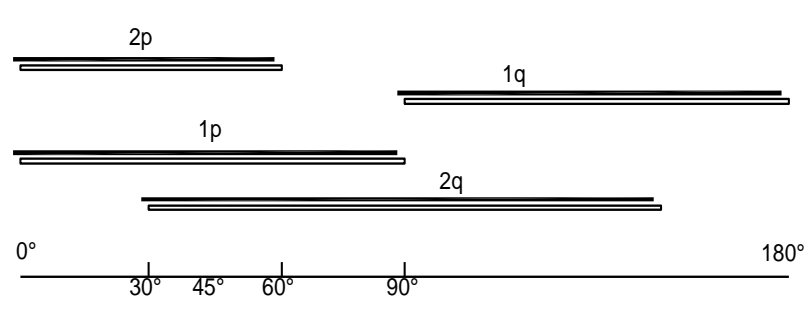

Fig.4. Calculation of $\mathrm{d}$ for 2-coverage

\section{Calculation of $d$ for 3-coverage:}

Here, the perimeter of sensor $\mathrm{M}$ is covered by nodes of type 1 , type 2 and type 3 .

Calculations of $\mathrm{d}_{(\mathrm{i}, \mathrm{j})}, \alpha_{1}$ and $\alpha_{2}$ for nodes of type 1 and type 2 are given in above case.

For node of type $3 \mathrm{~d}_{(\mathrm{i}, \mathrm{j})}=2 \mathrm{~d}$. So $\alpha_{3}=\arccos \left(\mathrm{d} / \mathrm{r}_{\mathrm{s}}\right)$.

For node $3 p \beta=0^{\circ}$. So arc of $\mathrm{M}$, perimeter covered by node $3 p$ is $\left[-\alpha_{3}, \alpha_{3}\right]$. Now from the figure we see that sensor $M$ is 3perimeter covered if arc of $\mathrm{M}$ perimeter covered by sensor $2 \mathrm{p}$ and sensor $3 q$ overlap.

Arc of M, perimeter covered by node $3 q$ is $\left[90^{\circ}-\alpha_{3}, 90^{\circ}+\alpha_{3}\right]$

Arc of $M$, perimeter covered by 2 pis $\left[-\alpha_{2}, \alpha_{2}\right]$.

So for 3 coverage $90^{\circ}-\alpha_{3}=\alpha_{2}$

This gives $\arcsin \left(\mathrm{d} / \mathrm{r}_{\mathrm{s}}\right)=\arccos \left(\mathrm{d} / 2 \mathrm{r}_{\mathrm{s}}\right)$.

Hence distance $d$ for 3 coverage $d=2 / \sqrt{ } 5 r_{s}$.

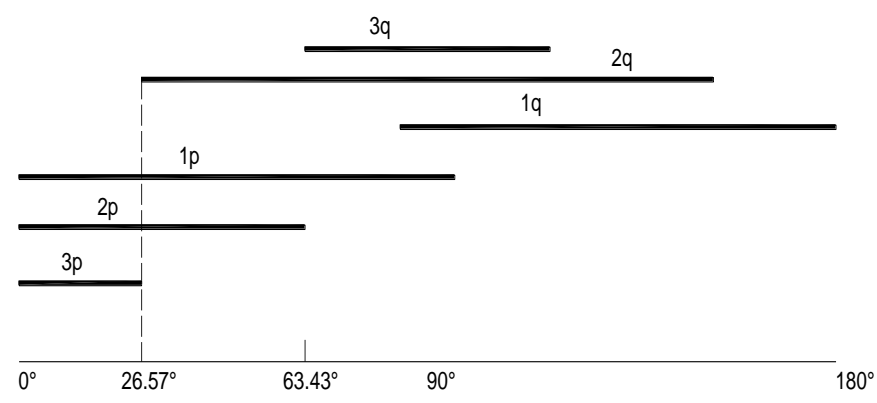

Fig 5.: Calculation of $d$ for 3-coverage

\section{Calculation of $\mathbf{d}$ for 4-coverage:}

Here, the perimeter of sensor $M$ is covered by nodes of type 1 ,type 2 ,type 3 ,type 4 and type 5 .

Calculations for $\mathrm{d}_{(\mathrm{i}, \mathrm{j})}, \alpha_{1}, \alpha_{2}$ and $\alpha_{3}$ for nodes of type 1 , type 2 and type 3 are already calculated.

For nodes of type 4 and type $5 \mathrm{~d}_{(\mathrm{i}, \mathrm{j})}=\sqrt{ } 5 \mathrm{~d}$

So $\alpha_{4}=\alpha_{5}=\arccos \left(\sqrt{5} \mathrm{~d} / 2 \mathrm{r}_{\mathrm{s}}\right)$.

For node $4 p \beta=\arctan (2)$. So arc of $M$, perimeter covered by node $4 p$ is $\left[\arctan (2)-\alpha_{4}, \arctan (2)+\alpha_{4}\right]$.

For node $4 p \beta=\arctan (0.5)$. So arc of $M$, perimeter covered by node $5 \mathrm{p}$ is $\left[\arctan (0.5)-\alpha_{5}, \arctan (0.5)+\alpha_{5}\right]$.

From the figure we see that node Mis 4 perimeter covered if the arc of $\mathrm{M}$, perimeter covered by node $4 \mathrm{p}$ and node $1 \mathrm{q}$ overlap.

Arc of M, perimeter covered by node $1 \mathrm{q}$ is $\left[135^{\circ}-\alpha_{1}, 135^{\circ}+\alpha_{1}\right]$. For 4 coverage $135^{\circ}-\alpha_{1}=\arctan (2)+\alpha_{4}$.

From this we get $\mathrm{d}=0.851 \mathrm{r}$.

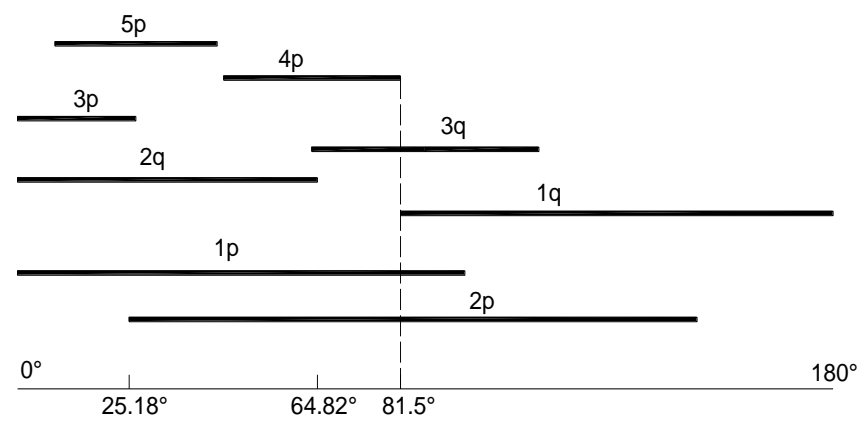

Fig 6. Calculation of $\mathrm{d}$ for 4-coverage

Calculation of $d$ for 5, 6, 7 coverage and above:

Here, the perimeter of sensor $\mathrm{M}$ is covered by nodes of all 5 types mentioned. We find that the distance between adjacent rows and columns are same for 5, 6 and 7 coverage using this scheme.

We get a 7 covered network when each node is 7 perimeter covered. Calculations for $\mathrm{d}_{(\mathrm{i}, \mathrm{j})}, \alpha_{1}, \alpha_{2}, \alpha_{3}, \alpha_{4}, \alpha_{5}$ are exactly the same as in previous cases. From figure node $M$ is 7 perimeter covered if the arc of $\mathrm{M}$, perimeter covered by node $1 \mathrm{~s}$ and node $2 q$ overlap.

For this $-45^{\circ}+\alpha_{1}=90^{\circ}-\alpha_{2}$. So $\mathbf{d}=2 \mathrm{r}_{\mathrm{s}} / \sqrt{ } 10$.

Using these values of $\mathbf{d}$ we deploy sensors for different levels of coverage and connectivity for the cases shown above.

For a generalised $n$ coverage $(n>4)$, we duplicate the 4 coverage deployment scheme $\mathrm{k}(=\mathrm{n} / 4)$ times and the $\mathrm{r}=(\mathrm{n}-\mathrm{k} * 4)$ coverage deployment scheme once. We use the 4 coverage deployment scheme repetitively because its gives the best results compared to other coverage levels.

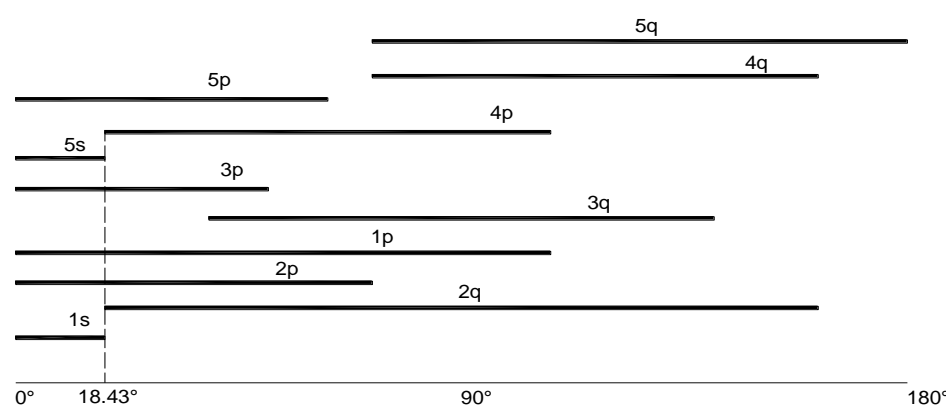

Fig.7: Calculation of $\mathrm{d}$ for 5,6-coverage

In the diagram of 7 coverage additional contribution are there due to nodes that are at a distance $3 \mathrm{~d}$ row-wise or columnwise and nodes that are at a distance $2 \mathrm{~d}$ row-wise and columnwise. These are not shown in the figure above and if we add them, the network will be 7 covered. The above diagram shows up to 6 coverage.

\section{Step 4: Deployment in the edge regions:}

In edges we evaluate the optimum distance $\mathbf{d}$ for 1-coverage and duplicate this scheme $\mathrm{n}$ times for $\mathrm{n}$ coverage problem. $\mathbf{d}$ is chosen so that the area covered by the strip (the region between the pair of horizontal lines in fig.8) is maximum. If $\mathrm{d}$ is the distance between adjacent nodes, the width of the strip is given by $2 * \sqrt{ }\left(\mathrm{r}_{\mathrm{s}}^{2}-\mathrm{d}^{2} / 4\right)$. 
So the area of strip will be maximum if the area $d .2 \sqrt{ }\left(r_{s}^{2}-d^{2} / 4\right)$ is maximum. For this we get $d=\sqrt{2} r_{s}$.

So we choose the width of the edge region as $\sqrt{ } 2 r_{s}$.

From coverage scheme in this case we duplicate the 1 coverage scheme $\mathrm{n}$ times.

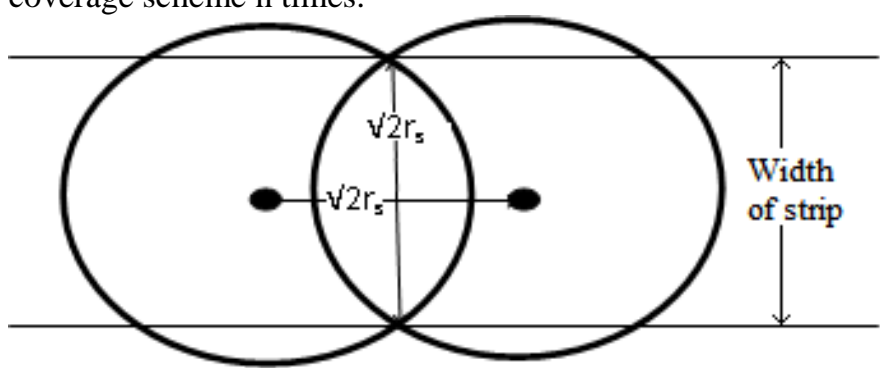

Fig.8: Deployment of sensors in edge regions

\section{SIMULATION RESULTS}

We compare the number of sensors required for our placement scheme and that for the interpolating scheme. The interpolating scheme is shown to be better than the naïve duplicate scheme [2] in most of the cases. We take an square area of $1000 \mathrm{~m}$ by $1000 \mathrm{~m}$ as our area of interest. Our scheme can be easily extended to any area of irregular shape. In that case we divide the area into square and rectangular shape areas. The communication range $\left(r_{c}\right)$ of the sensors is set to $10 \mathrm{~m}$, which is approximate to that specified in IEEE 802.15.4 standard for an indoor environment. We take 4 values of sensing range $\left(\mathrm{r}_{\mathrm{s}}\right)$ as $15 \mathrm{~m}, 11.55 \mathrm{~m}, 10 \mathrm{~m}$ and $8.04 \mathrm{~m}$. In all the cases we compare our scheme with the interpolating scheme. We compare 2,3,4,5,6,7 coverage schemes in the 4 cases.

TABLE 1

$\mathbf{r}_{\mathrm{s}}=\mathbf{1 5 m}, \mathbf{r}_{\mathbf{c}}=\mathbf{1 0 m}$
\begin{tabular}{|l|l|l|l|}
\hline $\mathrm{K}_{\text {(coverage) }}$ & $\mathrm{K}_{\text {(connectivity) }}$ & $\mathrm{N}_{\mathrm{s}}($ Our) & $\mathrm{N}_{\mathrm{s}}($ Their) \\
\hline 2 & 1 & 6778 & 7500 \\
\hline 3 & 1 & 7572 & 7500 \\
\hline 4 & 1 & 8144 & 10740 \\
\hline 5 & 5 & 11344 & 15000 \\
\hline 6 & 6 & 11532 & 15000 \\
\hline 7 & 7 & 11720 & 17890 \\
\hline
\end{tabular}

TABLE 2

$r_{\mathrm{s}}=11.55 \mathrm{~m}, \mathrm{r}_{\mathrm{c}}=10 \mathrm{~m}$

\begin{tabular}{|l|l|l|l|}
\hline $\mathrm{K}_{\text {(coverage) }}$ & $\mathrm{K}_{\text {(connectivity) }}$ & $\mathrm{N}_{\mathrm{s}}($ Our) & $\mathrm{N}_{\mathrm{s}}($ Their) \\
\hline 2 & 1 & 8826 & 9950 \\
\hline 3 & 1 & 10152 & 9950 \\
\hline 4 & 4 & 10992 & 14400 \\
\hline 5 & 5 & 19196 & 19000 \\
\hline 6 & 6 & 19444 & 19000 \\
\hline 7 & 7 & 19692 & 23300 \\
\hline
\end{tabular}

TABLE3

$\mathbf{r}_{\mathrm{s}}=\mathbf{1 0 m}, \mathbf{r}_{\mathbf{c}}=\mathbf{1 0 m}$
\begin{tabular}{|l|l|l|l|}
\hline $\mathrm{K}_{\text {(coverage) }}$ & $\mathrm{K}_{\text {(connectivity) }}$ & $\mathrm{N}_{\mathrm{s}}($ Our $)$ & $\mathrm{N}_{\mathrm{s}}($ Their) \\
\hline 2 & 2 & 10369 & 11070 \\
\hline 3 & 3 & 12952 & 13750 \\
\hline 4 & 4 & 14592 & 18750 \\
\hline 5 & 5 & 25445 & 24440 \\
\hline
\end{tabular}

\begin{tabular}{|l|l|l|l|}
\hline 6 & 6 & 25729 & 27010 \\
\hline 7 & 7 & 26013 & 32620 \\
\hline
\end{tabular}

TABLE 4

$\mathbf{r}_{\mathrm{s}}=\mathbf{8 m}, \mathbf{r}_{\mathbf{c}}=\mathbf{1 0 m}$
\begin{tabular}{|l|l|l|l|}
\hline $\mathrm{K}_{\text {(coverage) }}$ & $\mathrm{K}_{\text {(connectivity) }}$ & $\mathrm{N}_{\mathrm{s}}($ Our) & $\mathrm{N}_{\mathrm{s}}($ Their) \\
\hline 2 & 2 & 16072 & 14100 \\
\hline 3 & 3 & 20088 & 18240 \\
\hline 4 & 4 & 22417 & 24100 \\
\hline 5 & 5 & 39765 & 32200 \\
\hline 6 & 6 & 40113 & 34750 \\
\hline 7 & 7 & 40461 & 42150 \\
\hline
\end{tabular}

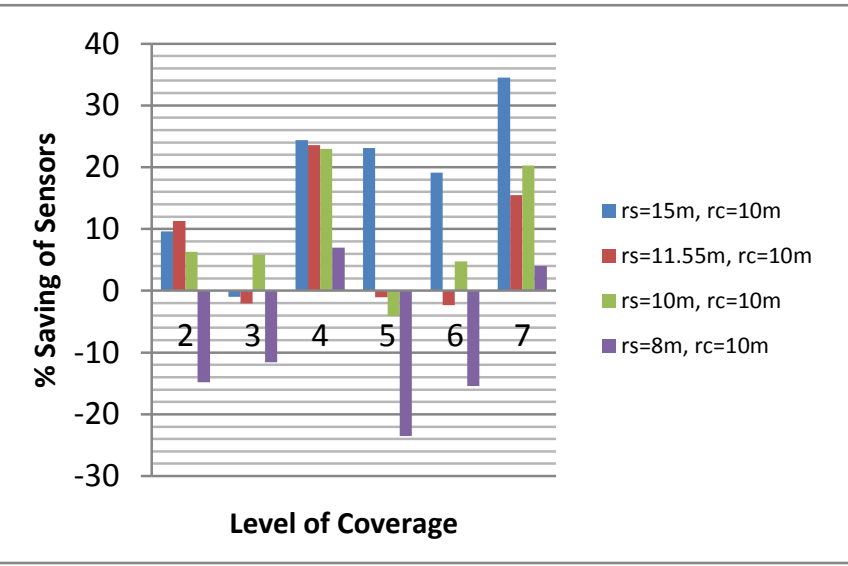

Fig.9: Percentage savings of sensors versus level of coverage

The abbreviations used are:

\begin{tabular}{|l|l|}
\hline Abbreviation & Meaning \\
\hline $\mathrm{K}_{\text {(coverage) }}$ & Level of coverage \\
\hline $\mathrm{K}_{\text {(connectivity) }}$ & Level of connectivity \\
\hline $\mathrm{N}_{\mathrm{s}}($ Our) & Number of sensors used in our scheme \\
\hline Ns( Their) & $\begin{array}{l}\text { Number of sensors used in Interpolating } \\
\text { scheme [2]. }\end{array}$ \\
\hline
\end{tabular}

From the comparisons we see that our scheme works better in most of the cases than the interpolating scheme. In some cases the interpolating scheme works better but that is marginally better in most instances. Moreover our scheme can be extended to achieve higher level of connectivity by adding only a few sensors.

Our scheme works especially well for 4 coverage and 7 coverage schemes. In all the comparisons given above for different relationships between $r_{s}$ and $r_{c}$ we see that our scheme always works better for these coverage levels compared to the interpolating scheme.

\section{CONCLUSIONS AND FUTURE WORK}

In this paper we have proposed a sensor deployment scheme for $\mathrm{k}_{\mathrm{s}}$ covered and $\mathrm{k}_{\mathrm{c}}$ connected network. There is no bound on the relationship between the communication distance and the sensing distance of the sensors, so this can be viewed as a generalised solution. We have also considered multilevel connectivity and multilevel coverage. 
In our work we assume a binary sensing model and take the sensing and communication ranges of the sensors to be circular in shape. As future work our work can be extended to probabilistic sensing model and also for sensing and communication regions of irregular shape. Energy saving protocols to increase the lifetime of sensor networks can also be implemented on the deployment scheme proposed in this paper and the utility of this scheme for lifetime enhancement can also be investigated.

\section{REFERENCES}

[1] C. F. Huang \& Y. C. Tseng, 'The Coverage Problem in a Wireless Sensor Network', WSNA'03, ACM, September, 2003.

[2] Y. C. Wang \& Y. C. Tseng, 'Distributed Deployment Schemes for Mobile Wireless Sensor Networks to Ensure Multi-level Coverage', IEEE Transactions On Parallel And Distributed Systems,2007

[3] Y. Zhou and Y. Fang, 'Securing Wireless Sensor Networks: A Survey', IEEE Communications, 2008

[4] X. Bai, S. Kumar, D. Xuan, Z. Yun, T. H. Lai, 'Deploying Wireless Sensors to Achieve Both Coverage and Connectivity' ,MobiHoc'06, ACM.

[5] J. R. Jiang and T. M. Sung, 'Energy-Efficient Coverage and Connectivity Maintenance for Wireless Sensor Networks', Journal of Networks, Aug'09.

[6] D. Dardari, A. Conti, C. Burrati, R. Verdone, 'Mathematical Evaluation of Environmental Monitoring Estimation Error through Energy-Efficient Wireless Sensor Networks', IEEE Transactions On Mobile Computing, July' 07.

[7] Z. Abrams, A. Goel, S. Plotkin, 'Set KCover Algorithms for Energy Efficient Monitoring in Wireless Sensor Networks', ACM, IPSN, April'04.

[8] Y. C. Wang, C. C. Hu, and Y. C. Tseng, "Efficient deployment algorithmsfor ensuring coverage and connectivity of wireless sensor networks,'Proc. IEEE First Int'l Conf. Wireless Internet (WICON '05), pp. 114-121, 2005.

[9] R. Kershner, "The number of circles covering a set," American J. Math.,vol. 61, pp. 665-671, Jul. 1939 